\title{
Long COVID and kidney disease
}

Sachin Yende (i] ${ }^{1,2 \bowtie ~ a n d ~ C h i r a g ~ R . ~ P a r i k h ~} \mathbb{1}^{3,4}$

Kidney involvement is common in patients with acute SARS-CoV-2 infection, and subclinical inflammation and injury may persist for many months, resulting in a progressive decline in kidney function that leads to chronic kidney disease. Continued research is imperative to understand these long-term sequelae and identify interventions to mitigate them. the relationship between COVID-19 and CKD is likely to be bidirectional

${ }^{1}$ Veteran Affairs Pittsburgh Healthcare System, Pittsburgh, PA, USA. ${ }^{2}$ Department of Critical Care Medicine, University of Pittsburgh, Pittsburgh, $P A, U S A$.

${ }^{3}$ Division of Nephrology, Department of Medicine, Johns Hopkins School of Medicine, Baltimore, MD, USA.

${ }^{4}$ Department of Epidemiology, Johns Hopkins School of Public Health, Baltimore, MD, USA.

凶e-mail:yendes@upmc.edu https://doi.org/10.1038/ s41581-021-00487-3
More than 180 million people have been diagnosed with COVID-19, and approximately 4 million have died worldwide. The short-term survival of these patients will improve as evidence-based therapies continue to be adopted and new treatments become available. Thus, the number of individuals who are at risk of poor longterm outcomes, known as long COVID, will continue to increase.

Approximately $28 \%$ of patients who are hospitalized with COVID-19 are diagnosed with acute kidney injury (AKI) and 9\% receive kidney replacement therapy ${ }^{1}$. Infection with SARS-CoV-2 has both direct and indirect effects that can lead to $\mathrm{AKI}^{2}$. Potential direct effects on the kidney include endothelial damage from viral entry, complement activation, local inflammation and collapsing glomerulopathy. However, indirect mechanisms that injure the kidney, such as sepsis, use of nephrotoxic medications, systemic inflammation, hypercoagulability and thromboembolic disease, might be more common than these direct effects. Although it is well known that AKI can lead to chronic kidney disease (CKD), little is known about the long-term effects of COVID-19 on the kidney.

Importantly, the COVID-19 pandemic is likely to have a long-term impact even on patients who have not been infected by the virus, owing to delays in care for chronic diseases, such as CKD, diabetes and hypertension ${ }^{3}$. This area is understudied but might have the largest impact at the population level and disproportionately affect vulnerable individuals, particularly those with low socioeconomic status and poor access to health care. Studies are urgently needed to investigate this area and confirm the importance of measures to catch up on preventive care for at-risk individuals.

What are the long-term effects of COVID-19 on the kidney? Three issues warrant consideration before we examine the current evidence. First, COVID-19 might affect the kidney in multiple ways and the contribution of these factors might vary over time. Many of the direct and indirect effects of the SARS-CoV-2 virus might persist during recovery after hospital discharge and could lead to repeat episodes of sepsis, recurrent AKI and increased risk of CKD (FIG. 1). Furthermore, a new diagnosis of diabetes mellitus and worsening of cardiovascular disease due to COVID-19 might delay recovery of AKI. Second, the relationship between COVID-19 and CKD is likely to be bidirectional. Mild CKD might increase the risk of COVID-19 and associated AKI, whereas increased severity of AKI might be associated with persistent kidney dysfunction, delayed recovery and/or need for long-term dialysis. Thus, understanding the effects of COVID-19 on the kidney will require use of study designs that account for this complex relationship and access to datasets in which kidney function is well characterized before and after diagnosis of COVID-19. Finally, whether the effects of COVID-19 are unique or similar to those seen in patients with other infections and sepsis is unclear. Initial reports suggest that many sequelae of COVID-19 are similar to those reported in survivors of sepsis and acute respiratory distress syndrome ${ }^{4}$.

A US study that used electronic health records from the Veterans Health Administration (VHA) to conduct a comprehensive assessment of long COVID reported that COVID-19 increased the risk of CKD and that this risk was highest among those who had severe illness ${ }^{5}$. Among patients who required hospitalization, adverse kidney manifestations, including urinary tract infections, AKI and CKD, occurred even after the first 30 days following diagnosis of COVID-19. Although the researchers used propensity matching, the possibility that these results are due to residual confounding cannot be excluded as some patients might have had subclinical CKD prior to hospitalization for COVID-19. Moreover, the risk of CKD among patients hospitalized with COVID-19 was similar to that of patients hospitalized with influenza.

The findings of the VHA study were similar to those of a study of patients with COVID-19 in China, which found that $35 \%$ of patients had reduced kidney function (estimated glomerular filtration rate (eGFR) $\left.<90 \mathrm{ml} / \mathrm{min} / 1.73 \mathrm{~m}^{2}\right) 6$ months after COVID-19 hospitalization. Interestingly, $13 \%$ of patients who did not have AKI during hospitalization showed a reduction in eGFR during follow-up ${ }^{6}$. Additional studies are underway in different populations with multiple control groups to better understand trajectories of kidney function during 


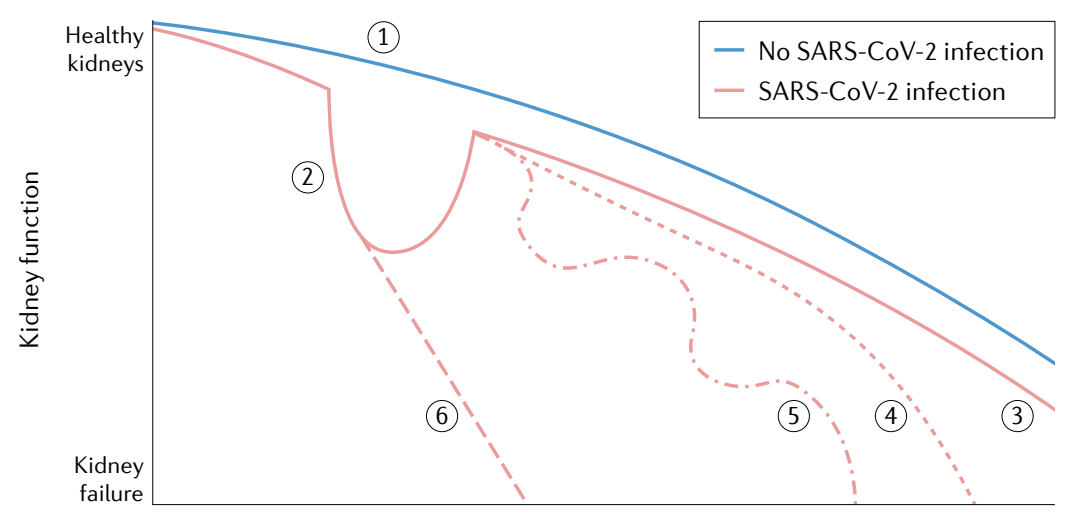

Time after SARS-CoV-2 infection

Fig. 1 | Conceptual model of trajectories of kidney function after SARS-CoV-2

infection. Patients with a high burden of frailty, chronic diseases, disability and immunosenescence are at increased risk of kidney disease and progression to kidney failure, and infection with SARS-CoV-2 can further increase this risk. Kidney function declines gradually over time even in the absence of SARS-CoV-2 infection (1), but patients who are hospitalized with COVID-19 might experience a rapid loss of kidney function resulting in acute kidney injury during the acute phase of the disease (2). Post-infection kidney function trajectories can vary and might include complete recovery (3), as indicated by no change in trajectory compared with patients who are not infected, slow progressive decline owing to subclinical inflammation (4), relapsing or recurrent acute kidney injury and hospitalizations (5) or incomplete recovery and rapidly progressive kidney disease (6). Adapted with permission from REF. ${ }^{10}$, Elsevier.

recovery in patients with COVID-19. These studies may provide additional insights.

Although in many patients serum creatinine returns to normal levels following AKI, the kidneys might not completely recover. Preclinical studies have demonstrated the development and persistence of inflammation, kidney fibrosis, abnormal kidney gene expression profiles and function deficits after ischaemic kidney injury, despite a return of serum creatinine concentrations to normal values. In COVID-19, these underlying pathophysiological mechanisms could be exaggerated together with worsening diabetes or hypertension control. Kidney disease progression in COVID-19 is likely multifactorial and might be driven by ongoing inflammation, intrinsic tubular injury or maladaptive repair, among other pathways. Novel kidney-specific plasma and urine biomarkers might help to discern the dominant underlying aetiologies and predict which patients are at highest risk of CKD after COVID-19 hospitalization?

To gain a better understanding of the effects of COVID-19 on the kidney, large integrated health-care systems and collaborations that enable rapid collection and analysis of clinical, laboratory and diagnostic data should continue to provide access to their databases for researchers who are investigating the long-term sequelae of COVID-19. For example, the National COVID Cohort Collaborative (N3C) has created a multisite collaborative learning health network with a scalable infrastructure, incorporating multi-organizational clinical data for COVID-19 analytics ${ }^{8}$. Translational studies in which patients with COVID-19 undergo data and biospecimen collection during hospitalization and recovery are key to understanding clinical and biological outcomes and investigate mechanisms. The NIH is planning to launch a multicentre, observational study of the post-acute sequelae of SARS-CoV-2 infection (PASC), with a special emphasis on cardiovascular and brain disorders ${ }^{9}$. The inclusion of novel and traditional measures of kidney function and injury in the PASC study will be important to investigate the mechanisms that underlie the development of CKD after COVID-19. Health services research is also needed to understand and mitigate health disparities that are potentially worsened by COVID-19. Leveraging electronic health records of the State Medicaid databases that provide care for vulnerable patients in the USA may be helpful.

Some changes in health care due to COVID-19 are likely to persist even after the pandemic. For example, the move towards the use of telemedicine, especially in the post-discharge setting, will continue. As telemedicine is an important tool for preventive and population health, understanding barriers to its use in some patient groups, such as those with low socioeconomic status, will be important. Finally, intervention studies to prevent incident CKD and progression of kidney disease will be paramount in reducing long-term morbidity and mortality after COVID-19.

In conclusion, the COVID-19 pandemic provides a unique opportunity to advance our understanding of how infection-associated AKI might worsen CKD and to develop novel therapies to delay progression of kidney disease. The pandemic has worsened disparities in health care, which may impact patients who are at-risk of or have CKD. Efforts to understand the effect of these disparities and identify steps to mitigate them should be an important focus of future research.

1. Silver, S. A. et al. The prevalence of acute kidney injury in patients hospitalized with COVID-19 infection: a systematic review and meta-analysis. Kidney Med. 3, 83-98 (2021).

2. Nadim, M. K. et al. COVID-19-associated acute kidney injury: consensus report of the 25th Acute Disease Quality Initiative (ADOl) Workgroup. Nat. Rev. Nephrol. 16, 747-764 (2020).

3. Findling, M. G., Blendon, R. J. \& Benson, J. M. Delayed care with harmful health consequences - reported experiences from national surveys during coronavirus disease 2019. JAMA Health Forum 1, e201463 (2020)

4. Prescott, H. C. et al. Understanding and enhancing sepsis survivorship. Priorities for research and practice. Am. J. Respir Crit Care Med. 200, 972-981 (2019).

5. Al-Aly, Z., Xie, Y. \& Bowe, B. High-dimensional characterization of post-acute sequelae of COVID-19. Nature 594, 259-264 (2021)

6. Huang, C. et al. 6-month consequences of COVID-19 in patients discharged from hospital: a cohort study. Lancet 397, 220-232 (2021).

7. Puthumana, J. et al. Biomarkers of inflammation and repair in kidney disease progression. J. Clin. Invest. 131, e 139927 (2021).

8. Haendel, M. A. et al. The National COVID Cohort Collaborative (N3C): rationale, design, infrastructure, and deployment. J. Am Med. Inform. Assoc. 28, 427-443 (2021).

9. National Institutes of Health. Research Opportunity Announcement OTA-21-015B. Post-acute sequelae of SARS-CoV-2 infection initiative: SARS-CoV-2 recovery cohort studies. NIH https://covid19 nih. gov/sites/default/files/2021-02/ PASC-ROA-OTA-Recovery-Cohort-Studies.pdf (2021).

10. Yende, S., Iwashyna, T. J. \& Angus, D. C. Interplay between sepsis and chronic health. Trends Mol. Med. 20, 234-238 (2014).

\section{Competing interests}

S.Y. has received funding from the Patient-centered Outcomes Research Institute (PCORI) and NIH. C.R.P. is supported by NIH grants, is a member of the advisory board of and owns equity in RenalytixAl, and serves as a consultant for Genfit and Novartis. The views expressed in this document do not represent the views of the Department of Veterans Affairs or funding agencies. 